\title{
PRODUÇÃO E ESTADO NUTRICIONAL DA SOJA EM FUNÇÃO DA CALAGEM
}

\author{
COUTINHO, Edson Luiz Mendes ${ }^{79}$ \\ COUTINHO NETO, André Mendes ${ }^{80}$ \\ ORIOLI JÚNIOR, Valdeci ${ }^{81}$ \\ SILVA, Alysson Roberto da ${ }^{82}$ \\ BUENO, Célia Regina Paes ${ }^{83}$
}

Recebido em: 2008-05-26

Aprovado em: 2008-08-12

ISSUE DOI: $10.3738 / 1982.2278 .79$

RESUMO: A maioria dos solos tropicais apresenta alta acidez, requerendo a aplicação de calcário para obter boas produtividades de soja. O objetivo deste trabalho foi estudar os efeitos da calagem no sistema solo-planta e na produtividade da soja cultivada no sistema plantio convencional. $\mathrm{O}$ experimento foi conduzido num solo argiloso (Latossolo Vermelho distroférrico), empregando-se as seguintes doses de calcário: 0 - 2,5 - 5,0 - 7,5 10,0 e 12,5 $\mathrm{Mg} \mathrm{ha}^{-1}$. A produtividade de grãos de soja aumentou com a calagem, sendo a produção mais elevada obtida com uma saturação por bases ao redor de $70 \%$. Em função da elevação do pH, houve aumento nas concentrações de N e P e redução nos teores de $\mathrm{Mn}$ e Zn nas folhas. As plantas daquelas parcelas que não receberam calcário exibiram sintomas de deficiência de $\mathrm{Mg}$, os quais estavam associados a uma concentração foliar de $1,7 \mathrm{~g} \mathrm{~kg}^{-1}$. O corretivo da acidez atuou também como fornecedor de $\mathrm{Mg}$ para as plantas.

Palavras-chave: Diagnose foliar. Glycine max. Micronutrientes. pH. Saturação por bases

\section{YIELD AND NUTRITIONAL STATUS OF SOYBEAN AS AFFECTED BY LIMING}

SUMMARY: Tropical soils are acid and, in order to overcome these conditions to grow soybean, lime is necessary. This experiment evaluated the effects of liming on some soil chemical attributes and on the yield, and nutritional status of soybean, under conventional till. The experiment was carried out on a Eutrustox soil, with the application of lime at rates of $0-2.5-5.0-7.5-10.0$, and $12.5 \mathrm{Mg} \mathrm{ha}^{-1}$. The soybean grain yield increased with lime levels, and maximum production was obtained at base saturation to approximately $70 \%$. The raising of soil $\mathrm{pH}$ resulted in significant increments in leaves concentration of $\mathrm{N}$ and $\mathrm{P}$, and reduced the contents of $\mathrm{Mn}$ and $\mathrm{Zn}$. In the plants those plots which did not receive lime, Mg deficiency symptoms were observed, which were associated with a $1.7 \mathrm{~g} \mathrm{~kg}^{-1}$ foliar content. The lime contributed to supply $\mathrm{Mg}$ to plants.

Keywords: Base saturation. Foliar diagnosis. Glycine max. Micronutrients. pH.

\footnotetext{
${ }^{79}$ Professor Titular. UNESP - Campus de Jaboticabal. E-mail: coutinho@fcav.unesp.br

${ }^{80}$ Acadêmico do Curso de Agronomia da FE/FAFRAM

${ }^{81}$ Mestrando em Agronomia (Produção Vegetal). UNESP - Campus de Jaboticabal.

${ }^{82}$ Doutor em Agronomia - Bunge Fertilizantes

${ }^{83}$ Prof $^{\mathrm{a}}$ Assistente Doutora. UNESP - Campus de Jaboticabal
} 


\section{INTRODUÇÃO}

Os solos da região tropical são bastante intemperizados e caracterizam-se pela alta acidez, presença de alumínio em níveis tóxicos e baixa fertilidade. Nessa situação, para o cultivo da soja, é necessária a correção da acidez e da construção da fertilidade do solo, através da aplicação de calcário e fertilizante, respectivamente.

Com relação à aplicação de calcário para a cultura da soja, os trabalhos realizados mostram de maneira geral, efeitos positivos desta prática na produção de grãos (NATALE; COUTINHO, 1994; MASCARENHAS et al. 1996; QUAGGIO et al. 1998; MIRANDA et al. 2005; NICOLODI et al. 2008).

Embora o conceito de correção da acidez do solo tenha evoluído nos últimos anos, os indicadores de acidez do solo e, principalmente, seus valores de referência para a recomendação de calcário, ainda se baseiam em experimentos realizados num passado relativamente distante, com a utilização de cultivares de soja com um patamar limitado, para os dias atuais, em termos de produtividade (MASCARENHAS et al. 1974; RAIJ et al. 1977; CAMARGO et al. 1982; QUAGGIO et al. 1982).

Nos últimos anos, vem se intensificando, em todo o Brasil, o uso do sistema plantio direto (SPD), atendendo aos apelos dos pesquisadores conservacionistas, a fim de se evitar a erosão do solo e conservá-lo, possibilitando assim uma agricultura sustentável. Na implantação do sistema, entretanto, é necessária uma eficiente correção da acidez, uma vez que, após a adoção do SPD, não haverá a possibilidade de incorporar o calcário e corrigir a acidez a maiores profundidades. Em função da produtividade atual dos cultivares de soja, uma nova definição dos valores de referência para a recomendação de calcário poderá ser requerido, principalmente em solos argilosos com alto poder tampão.

A necessidade, talvez, de utilização de maiores doses de calcário, exige cuidados, sendo importante nessa situação avaliar o estado nutricional das plantas, principalmente no tocante aos micronutrientes.

O objetivo deste trabalho foi estudar os efeitos da calagem no sistema solo-planta e na produtividade da soja cultivada num solo argiloso. 


\section{MATERIAL E MÉTODOS}

O experimento foi conduzido no município de Ribeirão Preto - SP, num solo classificado como Latossolo Vermelho distroférrico argiloso (EMBRAPA, 1999). Os principais atributos químicos iniciais do solo $(0-20 \mathrm{~cm})$ eram: $\mathrm{pH}\left(\mathrm{CaCl}_{2}\right) 4,1 ;$ M.O. $=28 \mathrm{~g}$

$\mathrm{dm}^{-3} ; \mathrm{P}($ resina $)=6 \mathrm{mg} \mathrm{dm}^{-3} ; \mathrm{K}=0,6 \mathrm{mmol}_{\mathrm{c}} \mathrm{dm}^{-3} ; \mathrm{Ca}=10 \mathrm{mmol}_{\mathrm{c}} \mathrm{dm}^{-3} ; \mathrm{Mg}=2 \mathrm{mmol}_{\mathrm{c}} \mathrm{dm}^{-3}$; $\mathrm{Al}=9 \mathrm{mmol}_{\mathrm{c}} \mathrm{dm}^{-3} ; \mathrm{H}+\mathrm{Al}=77 \mathrm{mmol}_{\mathrm{c}} \mathrm{dm}^{-3} ; \mathrm{CTC}=89,6 \mathrm{mmol}_{\mathrm{c}} \mathrm{dm}^{-3}$ e saturação por bases $(\mathrm{V})=14 \%$.

O experimento foi conduzido segundo o delineamento experimental em blocos casualizados, com seis tratamentos $\left(0 ; 2,5 ; 5,0 ; 7,5 ; 10,0\right.$ e $12,5 \mathrm{Mg} \mathrm{ha}^{-1}$ de calcário) e quatro repetições.

Cada parcela era constituída de seis linhas espaçadas de $0,50 \mathrm{~m}$, com comprimento de $8 \mathrm{~m}$, correspondendo uma área total de $24 \mathrm{~m}^{2}$ e uma área útil de $16 \mathrm{~m}^{2}$, pois se desprezou uma linha de cada lado da parcela que representaram a bordadura. As unidades experimentais foram separadas por carreadores de $2 \mathrm{~m}$.

Em agosto/2005 o calcário $(\mathrm{CaO}=46 \% ; \mathrm{MgO}=7 \% ; \mathrm{PRNT}=80 \%)$ foi aplicado a lanço na área total de cada parcela e incorporado com arado de discos e grade.

A semeadura foi realizada em novembro/2005, empregando-se sementes de soja [Glycine max (L.) Merrill cv. Conquista] previamente inoculadas com Bradyrhizobium específico (500 g de inoculante por $50 \mathrm{~kg}$ de sementes). Uma semana antes da semeadura, todos os tratamentos receberam uma adubação constante com $150 \mathrm{~kg} \mathrm{ha}^{-1}$ de $\mathrm{P}_{2} \mathrm{O}_{5}$ e $120 \mathrm{~kg}$ $\mathrm{ha}^{-1}$ de $\mathrm{K}_{2} \mathrm{O}$, tendo-se, respectivamente, como fontes, o superfosfato simples e o cloreto de potássio. Esses fertilizantes foram aplicados a lanço e incorporados com grade.

Para a avaliação do estado nutricional das plantas, no florescimento (estádio $\mathrm{R}_{2}$ ) coletou-se a $3^{\mathrm{a}}$ folha (com pecíolo) a partir do ápice da haste principal, de 30 plantas ao acaso na área útil de cada parcela. Nessa mesma época, foi realizada uma amostragem de solo na profundidade de 0-20 cm, com o objetivo de avaliar o $\mathrm{pH}\left(\mathrm{CaCl}_{2}\right)$ e os teores de $\mathrm{K}, \mathrm{Ca}$, $\mathrm{Mg}, \mathrm{Al}$ e $\mathrm{H}+\mathrm{Al}$.

Para a determinação das concentrações de macro e micronutrientes nas folhas, o material vegetal foi preparado e analisado segundo Bataglia et al. (1983). A análise química do solo foi realizada segundo Raij et al. (1987).

Os resultados foram submetidos à análise estatística conforme Banzatto; Kronka (1995). 


\section{RESULTADOS E DISCUSSÃO}

Verifica-se, na Tabela 1, que cinco meses após a aplicação das doses do corretivo da acidez, ocorreu um aumento significativo no $\mathrm{pH}$, na saturação por bases e nas concentrações de Ca e Mg no solo. De maneira oposta, a adição de calcário reduziu a acidez trocável ( $\mathrm{Al})$ e a acidez potencial $(\mathrm{H}+\mathrm{Al})$; os teores de $\mathrm{K}$ não foram alterados significativamente pela calagem.

Chama a atenção, o fato da dose de $5,0 \mathrm{Mg} \mathrm{ha}^{-1}$ de calcário que deveria, com base na análise química inicial do solo e através dos cálculos realizados segundo Raij et al. (1996), elevar a saturação por bases a valor próximo de 60\%. Para atingir valor próximo desse, entretanto, foi necessária uma dose de calcário $50 \%$ superior $\left(7,5 \mathrm{Mg}^{-1}\right)$. Resultados semelhantes foram obtidos em solos argilosos por Quaggio et al. (1982) e Miranda et al. (2005). Essa questão não deve ser atribuída a uma possível ineficiência do método da saturação por bases, mas sim à legislação brasileira de corretivos, por superestimar a reatividade das partículas de calcário retidas nas peneiras ABNT $\mathrm{n}^{\mathrm{os}} 20$ e 50 , conforme demonstrado por Natale; Coutinho (1994).

Tabela 1: Efeitos da calagem no $\mathrm{pH}$, saturação por bases (V) e nos teores de $\mathrm{K}, \mathrm{Ca}, \mathrm{Mg}, \mathrm{Al}$ e $\mathrm{H}+\mathrm{Al}$ no solo.

\begin{tabular}{ccccccccc}
\hline Calcário & $\begin{array}{c}\text { Produção } \\
\text { de grãos }\end{array}$ & $\mathrm{pH}$ & $\mathrm{Ca}$ & $\mathrm{Mg}$ & $\mathrm{K}$ & $\mathrm{Al}$ & $\mathrm{H}+\mathrm{Al}$ & $\mathrm{V}$ \\
\hline$------\mathrm{Mg} \mathrm{ha}^{-1}-------$ & & ------------- mmol $_{\mathrm{c}} \mathrm{dm}^{-3}$ & ---------------- & $---\%--$ \\
0 & 2,10 & 4,1 & 13 & 2 & 1,5 & 9 & 81,0 & 17 \\
2,5 & 2,80 & 4,5 & 26 & 4 & 1,6 & 4 & 72,1 & 31 \\
5,0 & 3,70 & 4,8 & 41 & 7 & 1,6 & 1 & 60,7 & 45 \\
7,5 & 4,10 & 5,1 & 55 & 11 & 1,6 & 0 & 51,9 & 57 \\
10,0 & 4,00 & 5,5 & 68 & 14 & 1,5 & 0 & 38,4 & 68 \\
12,5 & 4,05 & 5,8 & 79 & 15 & 1,5 & 0 & 28,0 & 77 \\
\hline Teste F & $11,36^{* *}$ & $94,25^{* *}$ & $15,39^{* * *}$ & $16,54^{* *}$ & $0,66 \mathrm{~ns}$ & $6,52^{* *}$ & $25,21^{* * *}$ & $65,32^{* *}$ \\
Regressão & $\mathrm{L}^{* *} \mathrm{Q}^{* *}$ & $\mathrm{~L}^{* *}$ & $\mathrm{~L}^{* *}$ & $\mathrm{~L}^{* *} \mathrm{Q}^{* *}$ & - & $\mathrm{L}^{* *}$ & $\mathrm{~L}^{* *}$ & $\mathrm{~L}^{* *} \mathrm{Q}^{* *}$ \\
\hline
\end{tabular}

** - significativo a $1 \%$.

$\mathrm{L}, \mathrm{Q}$ - regressão linear e quadrática, respectivamente.

Nota-se, através da Tabela 2, que a calagem alterou significativamente as concentrações de N, P, Mg, Mn e Zn nas folhas de soja. 
As plantas do tratamento sem calcário estavam deficientes em $\mathrm{N}$, apresentando concentrações do nutriente abaixo da faixa de suficiência sugerida por Ambrosano et al. (1996). Verificou-se, entretanto, que com o aumento das doses de calcário e conseqüentemente do $\mathrm{pH}$ do solo, ocorreram incrementos nas concentrações de $\mathrm{N}$ nas folhas (Figura 1). Deve-se destacar, que a adição da menor dose de calcário (2,5 $\left.\mathrm{Mg} \mathrm{ha}^{-1}\right)$ já foi suficiente para proporcionar teores adequados do macronutriente. Resultados semelhantes foram obtidos por Miranda et al. (2005).

Esse resultado pode ser atribuído à calagem proporcionar uma melhoria das condições do meio para o desenvolvimento da planta hospedeira e também para o crescimento do microrganismo fixador de $\mathrm{N}$ (REIS et al. 2006). Adicionalmente, o aumento do $\mathrm{pH}$ do solo pode ter promovido uma melhoria na disponibilidade de Mo (QUAGGIO et al. 1998), o que é importante para o funcionamento do sistema de fixação simbiótica de $\mathrm{N}$ nas leguminosas (DECHEN; NACHTIGALL, 2006).

Tabela 2: Efeitos da calagem nas concentrações de macro e micronutrientes nas folhas de soja.

\begin{tabular}{ccccccccccc}
\hline Calcário & $\mathrm{N}$ & $\mathrm{P}$ & $\mathrm{K}$ & $\mathrm{Ca}$ & $\mathrm{Mg}$ & $\mathrm{S}$ & $\mathrm{B}$ & $\mathrm{Cu}$ & $\mathrm{Mn}$ & $\mathrm{Zn}$ \\
\hline $\mathrm{Mg} \mathrm{ha}^{-1}$ & $--------------------\mathrm{g} \mathrm{kg}^{-1}------------------$ & \multicolumn{2}{c}{$---------\mathrm{mg} \mathrm{kg}^{-1}--------$} \\
0 & 35,0 & 2,2 & 21 & 10,0 & 1,7 & 2,2 & 46 & 9 & 97 & 37 \\
2,5 & 41,0 & 2,4 & 21 & 11,1 & 3,0 & 2,3 & 44 & 10 & 74 & 35 \\
5,0 & 45,1 & 2,9 & 19 & 10,6 & 3,3 & 2,2 & 42 & 9 & 65 & 34 \\
7,5 & 47,0 & 3,0 & 18 & 9,7 & 3,5 & 2,4 & 44 & 9 & 54 & 34 \\
10,0 & 46,8 & 2,9 & 20 & 10,3 & 3,4 & 2,3 & 46 & 10 & 42 & 30 \\
12,5 & 46,1 & 3,1 & 18 & 10,9 & 3,6 & 2,3 & 43 & 10 & 31 & 27 \\
\hline Teste F & $9,23^{* *}$ & $5,61^{* *}$ & $0,99 \mathrm{~ns}$ & $0,72 \mathrm{~ns}$ & $4,94^{* *}$ & $0,36 \mathrm{~ns}$ & $0,21 \mathrm{~ns}$ & $0,16 \mathrm{~ns}$ & $12,51^{* *}$ & $9,11^{* *}$ \\
Regressão & $\mathrm{L}^{*} \mathrm{Q}^{* *}$ & $\mathrm{~L}^{*} \mathrm{Q}^{* *}$ & - & - & $\mathrm{L}^{* *} \mathrm{Q}^{* *}$ & - & - & - & $\mathrm{L}^{* *}$ & $\mathrm{~L}^{* * *}$
\end{tabular}

$*$, ** - significativo a 5 e $1 \%$, respectivamente.

$\mathrm{L}, \mathrm{Q}$ - regressão linear e quadrática, respectivamente

De maneira análoga ao $\mathrm{N}$, em condições de baixo $\mathrm{pH}$ do solo, as plantas apresentavam-se carentes em P (Tabela 2 e Figura 1), segundo Ambrosano et al. (1996).

$\mathrm{O}$ aumento do $\mathrm{pH}$ do solo através da calagem, induzindo uma maior disponibilidade de P para as plantas, também foi observado por Raij; Quaggio (1990). De acordo com Sousa et al. (2007), o aumento do pH do solo além de diminuir a adsorção do P nos óxidos de Fe e Al, neutraliza o Al trocável, proporcionando um maior desenvolvimento das raízes, o que 
possibilita uma exploração de um maior volume de solo. Nesse contexto, observa-se na Tabela 1 que a adição de 5,0 $\mathrm{Mg} \mathrm{ha}^{-1}$ de calcário praticamente neutralizou o $\mathrm{Al}$ trocável.

Com relação às concentrações de $\mathrm{Mg}$ nas folhas, como era esperado, à medida que se aumentaram as doses de calcário, os teores desse nutriente também se elevaram, alcançando concentrações de até $3,6 \mathrm{~g} \mathrm{~kg}^{-1}$ (Tabela 2).

É importante mencionar, que o teor inicial de $\mathrm{Mg}$ no solo era baixo (QUAGGIO, 2000). Em função disso, foram verificados nas plantas do tratamento que não recebeu a aplicação do corretivo da acidez, no estádio de florescimento, sintomas típicos de deficiência desse cátion divalente. Essa observação foi confirmada através dos teores de $\mathrm{Mg}$ nas folhas, os quais encontravam-se abaixo da faixa de suficiência sugerida por Ambrosano et al. (1996). Destaca-se que a deficiência era corrigida com a aplicação da menor dose de calcário $(2,5 \mathrm{Mg}$ ha $^{-1}$ ). Raij et al. (1977) também verifica sintomas de carência de Mg em soja cultivada num solo com teor de $\mathrm{Mg}=10 \mathrm{mmol}_{\mathrm{c}} \mathrm{dm}^{-3}$.
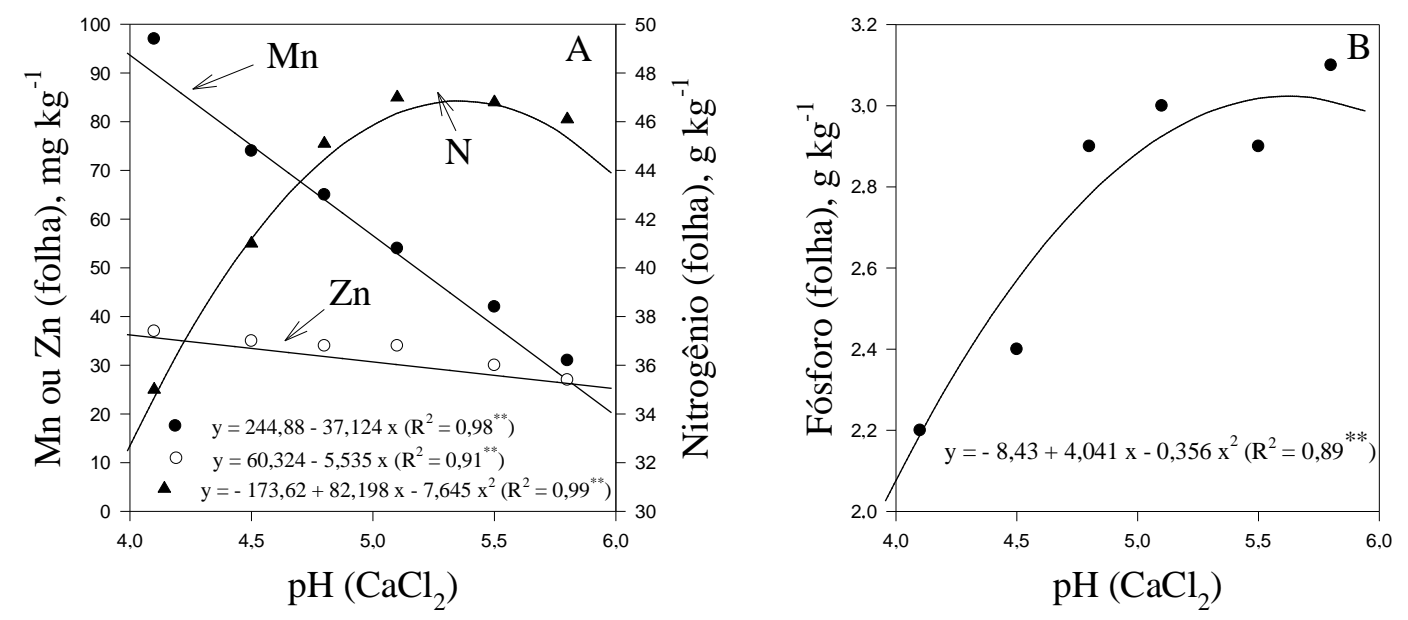

Figura 1: Relação entre o pH do solo e as concentrações de N, Mn, Zn (A) e P nas folhas de soja (B).

A elevação dos valores de pH através da aplicação de doses crescentes de calcário promoveu uma redução significativa nas concentrações de $\mathrm{Mn}$ e $\mathrm{Zn}$ nas folhas de soja (Figura 1). Essa diminuição na disponibilidade de Mn e Zn no solo, devido ao aumento do $\mathrm{pH}$, pode ser atribuída à redistribuição desses cátions do reservatório trocável (forma fitodisponível) para os reservatórios óxidos de ferro e alumínio cristalino e pouco cristalino (formas menos fitodisponíveis), conforme observaram Chimello (2001) e Consolini; Coutinho (2004). 
O efeito depressivo da calagem sobre a disponibilidade de alguns micronutrientes para a soja é preocupante, em função de freqüentemente deficiências terem sido associadas ao excesso de calcário (MASCARENHAS et al. 1988; NOVAIS et al. 1989). No presente trabalho, não obstante ter se empregado doses consideradas elevadas de calcário (até $12,5 \mathrm{Mg}$ $\left.\mathrm{ha}^{-1}\right)$, atingindo-se $\mathrm{pH}\left(\mathrm{CaCl}_{2}\right)$ próximo a 6,0 , não se observou problemas nutricionais com os micronutrientes. As concentrações de $\mathrm{B}, \mathrm{Cu}, \mathrm{Mn}$ e $\mathrm{Zn}$ estavam dentro da faixa considerada adequada por Ambrosano et al. (1996).

Também, as concentrações de K, Ca e S nas folhas estiveram dentro da faixa de teores considerados adequados por Ambrosano et al. (1996) e não foram influenciadas significativamente pela calagem. Corroborando resultados de Raij et al. (1977), chama a atenção o fato do corretivo da acidez não ter atuado como fonte de $\mathrm{Ca}$, uma vez que as concentrações desse cátion apresentaram-se na faixa considerada adequada, mesmo nas plantas que não receberam calcário, indicando que o solo e o superfosfato simples $(26 \%$ de $\mathrm{CaO}$ ), empregado na adubação de semeadura, continham esse nutriente em quantidades suficientes para a soja.

Observa-se na Tabela 1, que a aplicação de calcário aumentou significativamente a produtividade de grãos de soja. Nota-se, entretanto, que o incremento na produção praticamente estabilizou a partir da dose de 7,5 $\mathrm{Mg} \mathrm{ha}^{-1}$. Esses dados demonstram a importância da correção da acidez do solo para a cultura da soja, como verificaram Natale; Coutinho (1994), Mascarenhas et al. (1996), Quaggio et al. (1998), Miranda et al. (2005) e Nicolodi et al. (2008).

Nesse tipo de estudo, é bastante interessante, procurar relacionar a produção com os indicadores de acidez do solo.

Nesse sentido, verifica-se na Figura 2 que as maiores produtividades de grãos de soja estiveram associadas a uma saturação por bases de aproximadamente $70 \%$ e $\mathrm{pH}\left(\mathrm{CaCl}_{2}\right)$ 5,5. 

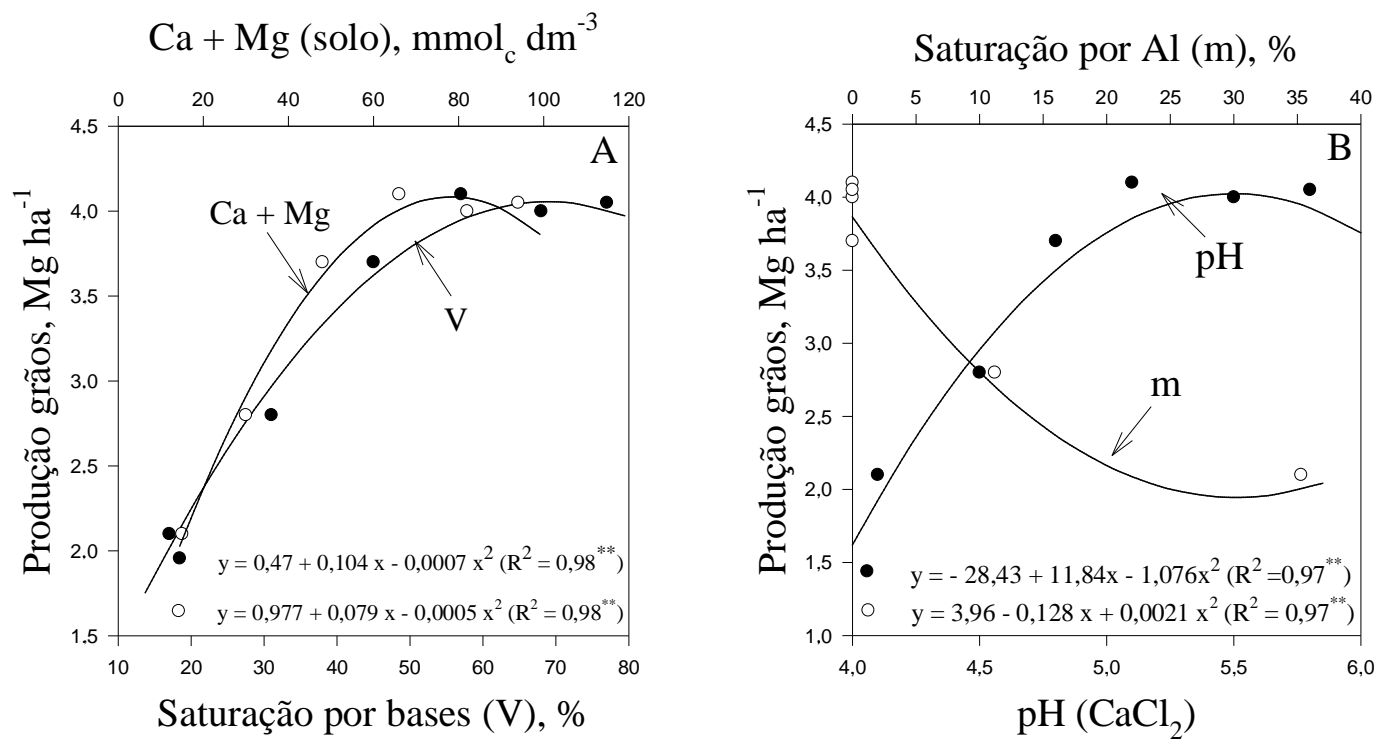

Figura 2: Relação entre a produção de grãos de soja e a saturação por bases, teores de $\mathrm{Ca}+$ $\mathrm{Mg}$ no solo (A), pH e saturação por $\mathrm{Al}(\mathrm{B})$.

Esse valor determinado de saturação por bases é superior aos recomendados para a cultura da soja no estado de São Paulo e para a região dos cerrados (AMBROSANO et al., 1996; EMBRAPA, 2006). Esse resultado comprova a hipótese inicial, sobre a necessidade de uma nova definição dos valores de referência para a recomendação de calcário, em vista dos cultivares atuais de soja serem mais produtivos.

Na Figura 2, nota-se ainda, o fato da maior produção de soja ter sido obtida com uma soma dos cátions $\mathrm{Ca}+\mathrm{Mg}$ no solo ao redor de $79 \mathrm{mmol}_{\mathrm{c}} \mathrm{dm}^{-3}$, valor bastante superior ao recomendado para o cultivo dessa leguminosa na região Centro-Oeste do Brasil (EMBRAPA, 2006). Por outro lado, quando se relaciona a saturação por alumínio e a produção (Figura 2), a máxima produtividade não é conseguida, o que indica que mesmo quando os valores de $\mathrm{Al}$ trocável não são detectáveis na análise química do solo, há ainda a possibilidade de incrementos na produção de grãos pela adição de mais calcário. Resultados semelhantes foram obtidos por Raij et al. (1977).

\section{CONCLUSÃO}

A calagem aumentou significativamente a produtividade de grãos de soja, estando as maiores produtividades associadas a um valor $\mathrm{pH}\left(\mathrm{CaCl}_{2}\right)$ 5,5, a uma saturação por bases ao redor de $70 \%$ e concentrações no solo de $\mathrm{Ca}+\mathrm{Mg}=79 \mathrm{mmol}_{\mathrm{c}} \mathrm{dm}^{-3}$. 
Em função da elevação do pH, houve aumento nas concentrações de $\mathrm{N}$ e $\mathrm{P}$ e redução nos teores de Mn e $\mathrm{Zn}$ nas folhas. O corretivo da acidez atuou também como fornecedor de Mg para as plantas.

\section{REFERÊNCIAS}

AMBROSANO, E. J. et al. Leguminosas e oleaginosas. In: RAIJ, B. van; CANTARELLA, H.; QUAGGIO, J. A.; FURLANI, A. M. C. (eds.). Recomendações de adubação e calagem para o Estado de São Paulo, 2.ed. Campinas: Instituto Agronômico \& Fundação IAC, 1996. p.189-191.

BANZATTO, D. A.; KRONKA, S. N. Experimentação agrícola. Jaboticabal, FUNEP, 1995. $247 p$.

BATAGLiA, O. C. et al. Métodos de análise química das plantas. Campinas, Instituto Agronômico, 1983. 48p. (Boletim Técnico, 78).

CAMARGO, A. P.et al. Efeito da calagem nas produções de cinco cultivos de milho, seguidos de algodão e soja. Pesquisa Agropecuária Brasileira, v.17, p.1007-1012, 1982.

CHIMELLO, M. A. Efeito do pH do solo e da aplicação de manganês na distribuição e disponibilidade do micronutriente para a soja (Glycine max.(L.) Merrill). 2001. 107f. Dissertação (Mestrado) - Faculdade de Ciências Agrárias e Veterinárias, Universidade Estadual Paulista. Jaboticabal.

CONSOLINI, F; COUTINHO, E. L. M. Efeito da aplicação de Zn e do pH do solo na disponibilidade do micronutriente. Acta Scientiarum. Agronomy, v. 26, p.7-12, 2004.

DECHEN, A R; NACHTIGALL, G. R. Micronutrientes. In: FERNANDES, M.S. (Ed.). Nutrição mineral de plantas. Viçosa: Sociedade Brasileira de Ciência do Solo, 2006. p.327354.

EMBRAPA-EMPRESA BRASILEIRA DE PESQUISA AGROPECUÁRIA. Centro Nacional de Pesquisa de Soja. Tecnologias de produção de soja - Região Central do Brasil - 2007. Londrina: Embrapa Soja, Embrapa Cerrados, Embrapa Agropecuária Oeste, 2006. 225 p.

EMBRAPA-EMPRESA BRASILEIRA DE PESQUISA AGROPECUÁRIA. Centro Nacional de Pesquisa de Solos. Sistema brasileiro de classificação de solos. Brasília: Embrapa Produção de informação, 1999. 412p.

MASCARENHAS, H. A. A et al. Efeitos da calagem aplicada de uma só vez ou parcelada, na produção de soja. Bragantia, v.33, p.58-61, 1974.

MASCARENHAS, H.A.A.et al. Deficiência de boro em soja. Bragantia, v.47, p.325-331, 1988. 
MASCARENHAS, H. A. A.et al. Efeito da calagem sobre a produtividade de grãos, óleo e proteína em cultivares precoces de soja. Scientia Agrícola, v.53, p.164-172, 1996.

MIRANDA, L. N. et al. Utilização de calcário em plantio direto e convencional de soja e milho em Latossolo Vermelho. Pesquisa Agropecuária Brasileira, v.40, p.563-572, 2005.

NATALE, W; COUTINHO, E. L. M. Avaliação da eficiência agronômica de frações granulométricas de um calcário dolomítico. Revista Brasileira de Ciência do Solo, v.18, p.55-62, 1994.

NICOLODI, M; ANGHINONI, I; GIANELLO, C. Indicadores da acidez do solo para recomendação de calagem no sistema plantio direto. Revista Brasileira de Ciência do Solo, v.32, p.237-247, 2008.

NOVAIS, R. F. et al. Deficiência de manganês em plantas de soja cultivadas em solos de cerrado. Revista Brasileira de Ciência do Solo, v.13, p.199-204, 1989.

QUAGGIO, J. A. Acidez e calagem em solos tropicais. Campinas: Instituto Agronômico, 2000. $111 \mathrm{p}$.

QUAGGIO, J. A.et al. Isoquantas de produtividade de soja e sorgo para níveis de calagem e molibdênio. Revista Brasileira de Ciência do Solo, v.22, p.337-344, 1998.

QUAGGIO, J. A; MASCARENHAS, H. A. A; BATAGLIA, O. C. Resposta da soja à aplicação de doses crescentes de calcário em Latossolo Roxo distrófico de cerrado. II - Efeito residual. Revista Brasileira de Ciência do Solo, v.6, p.113-118, 1982.

RAIJ, B. et al. Efeito de níveis de calagem na produção de soja em solo de cerrado. Revista Brasileira de Ciência do Solo, v.1, p.28-31, 1977.

RAIJ, B. et al. Recomendações de adubação e calagem para o Estado de São Paulo, 2.ed. Campinas: Instituto Agronômico \& Fundação IAC, 1996. 285 p.

RAIJ, B. van.; QUAGGIO, J. A. Extractable phosphorus availability indexes as affected by liming. Communications in Soil Science and Plant Analysis, v.21, p.1267-1276, 1990.

RAIJ, B. et al. Análise química do solo para fins de fertilidade. Campinas, Fundação Cargill, 1987. 170p.

REIS, V. M. et al. Fixação biológica de nitrogênio simbiótica e associativa. In: FERNANDES, M.S. (Ed.). Nutrição mineral de plantas. Viçosa: Sociedade Brasileira de Ciência do Solo, 2006. p.153-174.

SOUSA, D. M. G. et al. Fertilidade do solo. Viçosa: Sociedade Brasileira de Ciência do Solo, 2007. p.205-274. 